\title{
REPRESENTATIONS OF ALGEBRAIC GROUPS
}

\author{
ROBERT STEINBERG*
}

To Professor Richard Brauer on the occasion of his 60 th birthday

\section{$\S 1$. Introduction}

Our purpose here is to study the irreducible representations of semisimple algebraic groups of characteristic $p \neq 0$, in particular the rational representations, and to determine all of the representations of corresponding finite simple groups. (Each algebraic group is assumed to be defined over a universal field which is algebraically closed and of infinite degree of transcendence over the prime field, and all of its representations are assumed to take place on vector spaces over this field.)

To state our first principal result, we observe that relative to a Cartan decomposition of a semisimple algebraic group, there is described in $\S 5$ below (in a somewhat more general context) a standard way of converting an isomorphism on the universal field into one on the group, and that relative to a choice of a set $S$ of simple roots, an irreducible rational projective representation of the group is characterized by a function from $S$ to the nonnegative integers, to be called, together with the corresponding function on the Cartan subgroup of the decomposition, the high weight of the representation [13, Exp. 14 and 15].

1.1 THEOREM. Let $G$ be a semisimple algebraic group of characteristic $p \neq 0$ and rank $l$, and let $\Re$ denote the set of $p^{l}$ irreducible rational projective representations of $G$ in each of which the high weight $\lambda$ satisfies $0 \leq \lambda(\boldsymbol{a}) \leq(p-1)$ $(a \in S)$. Let $\alpha_{i}$ denote the automorphism $t \rightarrow t^{p^{i}}$ of the universal field as well as the corresponding automorphism (see $\S 5$ ) of $G$, and for $R \in \Re$ let $R^{\alpha_{i}}$ denote the composition of $\alpha_{i}$ and $R$. Then every irreducible rational projective representation of $G$ can be written uniquely as $\Pi_{i=0}^{\infty} R_{i}^{\alpha_{i}}$ (weak tensor product, $R_{i} \in \mathfrak{R}$ ).

Received May 21, 1962.

* This research was supported by the Air Force Office of Scientific Research. 
Conversely, every such product yields an irreducible rational projective representation of $G$.

This follows from 6.1 below. We need only remark here that there is no corresponding phenomenon for groups of characteristic 0 , since then the identity is the only rational field automorphism and the tensor product of two rational representations is never irreducible unless one of them is one-dimensional. Related to 1.1 is the following conjecture for which there is much evidence and for which a proof for the group of type $A_{1}$ would go a long way.

1.2 Conjecture. If $G$ and $\Re$ are as in 1.1 and $R$ is an irreducible, not necessarily rational, projective representation of $G$, there exist distinct isomorphisms $\beta_{i}$ of the universal field into itself and corresponding representations $R_{i}$ in $\Re$ such that $R=\Pi R_{i}^{\beta^{2}}$ (see $\S 5$ for the definition of $R_{i}^{\beta i}$ ).

That the above product is always irreducible follows from 5.1 below.

Our second main result applies to naturally defined finite simple subgroups of the groups considered above. These include all the "finite simple algebraic groups" (those made up of the rational points of simple algebraic groups suitably defined over finite fields), that is (see Hertzig [8]), the groups considered by Chevalley [3] and those considered by Hertzig [8], Tits [24, 25] and the author $[19,20]$, and also include the nonalgebraic groups considered by Suzuki [22] and Ree [11], all the known finite simple groups other than the cyclic, alternating and Mathieu groups.

1.3 Theorem. If $G$ is a finite simple algebraic group and the rational field has $q=p^{n}$ elements, then every irreducible projective representation is the restriction of a rational representation of the corresponding infinite algebraic group. If the rank is $l$, the number of such representations is $q^{l}$. Each has a high weight $\lambda$ for which $0 \leq \lambda(a) \leq q-1 \quad(a \in S)$.

Here we also have the product representation of 1.1 with the upper limit $n$ in place of $\infty$ (see 7.4 and 9.3). For the nonalgebraic finite groups mentioned above there is a corresponding result ( 12.2 below), but the relevant representations of the containing infinite algebraic groups are those that satisfy the further condition: $\lambda(a)=0$ if $a$ is long; hence their number is $q^{l / 2}$. A gap in our development is that for finite odd-dimensional unitary groups and finite 
Ree groups of type $G_{2}$ we have established these results, and also the following (see 8.1, 8.2, 9.6 and 12.5) only for ordinary representations, not for projective representations.

1.4. Theorem. Each of the finite groups above, algebraic or not, has an irreducible (ordinary) representation of dimension equal to the order of a pSylow subgroup. No other irreducible (projective or ordinary) representation has as high a dimension.

Among the subsidiary results below, we consider the character of this highest representation $(8.4,9.6,11.3)$, and present in $\S \S 10$ and 11 some results related to those rather special isogenies which give rise to the existence of the groups of Suzuki and Ree.

In addition to [13], to which frequent references will be made, earlier work related to our results is as follows. In [2] Brauer and Nesbitt determine the irreducible representations of finite groups of type $S L(2)$ and prove the appropriate tensor product theorem, while in [13, Exp. 20] Chevalley does the same for rational representations of the corresponding infinite groups. In [10] Mark considers the finite groups of type $S L(3)$, while in [27] Wong considers groups of type $S L(l+1)$ and $S p(l)$ and proves $1.1,1.3$ and 1.4 for ordinary representations. His methods, however, are quite different from ours, and are not readily extendable to the other types of groups. Our methods are closely related to those of Curtis in [4] where the representations of $\Re$ in 1.1 are constructed by infinitesimal methods and in [5] where they are shown to remain irreducible on restriction to the corresponding finite Chevalley groups (under the assumption $p>7$, which can easily be removed).

\section{§ 2. Classical Lie algebras}

Let $\mathrm{g}_{c}$ be a simple Lie algebra over the complex field $C$, $\mathfrak{h}_{c}$ a Cartan subalgebra, $\sum$ the (ordered) system of roots relative to $\mathfrak{h}_{c}, S$ the set of simple positive roots, and for each pair $r, s$ of roots, set $c_{r s}=2(r, s) /(s, s)$, and define $p_{r s}$ to be 0 if $r+s$ is not a root, otherwise to be the least positive integer $p$ for which $r-p s$ is not a root. Then Chevalley [3, p. 24] has shown that there exists a generating set $\left\{X_{r}, H_{r} \mid r \in \sum\right\}$ such that the equations of structure of $g_{c}$ are: 
2.1. $H_{-r}=-H_{r} \quad\left(r \in \sum\right)$.

2.2. $H_{r+i s}=H_{r}+H_{s}$ if $i$ is a positive integer and $r+i$ and $r$ have the maximum root length.

2.3. $\left[H_{r}, H_{s}\right]=0 \quad\left(r, s \in \sum\right)$.

2.4. $\left[H_{r}, X_{s}\right]=c_{s r} X_{s} \quad\left(r, s \in \sum\right)$.

2.5. $\left[X_{r}, X_{-r}\right]=H_{r} \quad\left(r \in \sum\right)$.

2.6. $\left[X_{r}, X_{s}\right]= \pm p_{r s} X_{r+s} \quad\left(r, s \in \sum, r+s \neq 0\right)$.

Let $g$ and $\mathfrak{h}$ denote the algebras obtained by shifting the coefficients to an arbitrary field $K$ of characteristic $p$. Then $X_{r}$ and $H_{r}$ shall be considered to belong to $g$ but the subscript $r$ shall continue to denote an element of $\Sigma$. For the algebras just constructed, Curtis [4] has developed a theory of irreducible representations quite analogous to the classical theory in characteristic 0 . Although he states and proves his results under the assumption that $K$ is algebraically closed and $p>7$, his proofs can be modified to apply to the present situation. We recall that a representation $\rho$ of $g$ is restricted if $\rho\left(X_{r}\right)^{p}=0$ and $\rho\left(H_{r}\right)^{p}=\rho\left(H_{r}\right)$ for each root $r$.

2.7 Curtis. With $\mathrm{g}$ as above, every irreducible restricted g-module $M$ contains a nonzero element $v_{+}$, uniquely determined to within multiplication by a scalar, such that $X_{r} v_{+}=0$ if $r$ is positive, and there exist integers $\lambda(a)$, $0 \leq \lambda(a) \leq p-1$, such that $H_{a} v_{+}=\lambda(a) v_{+}(a \in S)$. Inequivalent modules yield distinct sequences $\lambda(a)$, and all sequences are realized. Thus there are $p^{l}$ modules for an algebra of rank $l$.

Here and elsewhere in the paper, " $g$-module" means vector space over the algebraic closure $\bar{K}$ of $K$ on which $\bar{K}$ and $g$ act according to the usual rules, "irreducible" means absolutely irreducible, and $\mathfrak{M}$ denotes the $p^{l}$ modules given by 2.7. As is easily seen, the modules of $\mathfrak{M}\left(g_{\bar{K}}\right)$ may be viewed as extensions of those of $\mathfrak{M}\left(g_{K}\right)$, or equivalently, the latter as restrictions of the former. For each $M \in \mathfrak{M}, v_{\dot{+}}$ is called a high vector and the linear function $\lambda$ on $\mathfrak{h}$ defined by $\lambda\left(H_{a}\right)=\lambda(a)$ the high weight of $M$. Further for a positive root $r=\sum n(a) a \quad(a \in S)$, we set ht $r=\sum n(a)$, the height of $r$, then order the positive roots $r_{1}, r_{2}, \ldots, r_{m}$ in a manner consistent with heights (if ht $r_{i}<$ ht $r_{j}$, then $i<j$ ), and for the monomial

$$
v=X_{-r_{m}}^{i_{m}} \cdots X_{-r_{2}}^{i_{2}} X_{-r_{1}}^{i_{1}} v_{+} \quad\left(0 \leq i_{k} \leq p-1\right)
$$


set ht $v=-\sum i_{k}$ ht $r_{k}$, and finally call $w$ homogeneous of height $n$ if it is a linear combination of monomials of height $n$. We recall that a basis for $M$ can be selected from the monomials.

2.9 Lemma. Nonzero vectors of different heights are linearly independent.

Proof. Given a relation $v_{0}+v_{1}+\cdots+v_{d}=0$ with $v_{i}$ of height $-i$, we prove by induction on $d$ that each $v_{i}$ is 0 . If $d=0$, this is clear.

Assume $d>0$. If $r$ is any positive root, $X_{r} v_{0}+X_{r} v_{1}+\cdots+X_{r} v_{d}=0$, and since $X_{r} v_{i}$ is higher than $v_{i}$, the induction assumption yields $X_{r} v_{d}=0$. Thus by 2.7, $v_{d} \in K v_{+}$, and since the algebra generated by those $X_{-r}$ for which $r>0$ acts nilpotently on $M$ because $M$ is restricted, $v_{d}=0$. Then each $v_{i}$ is 0 by the induction assumption.

\section{§ 3. Classical algebraic groups}

Now set $x_{r}(t)=\exp a d t X_{r}\left(t \in K, r \in \sum\right)$, and let $G$ (this is $G^{\prime}$ in [3]) denote the group generated by all of these automorphisms. With 4. exceptions $[3$, p. 63$]$, which we henceforth exclude, $G$ is simple. In $G$ there are commutator relations $[3$, p. 36$]$ :

$$
\text { 3.1. }\left(x_{r}(t), x_{s}(u)\right)=\Pi x_{i r+j s}\left(C_{i j, r s} t^{i} u^{j}\right) \quad\left(r, s \in \sum, r+s \neq 0\right) .
$$

Here the product is taken over all positive integers $i, j$ for which $i r+j s$ is a root, the terms being arranged in some fixed, but arbitrary, order, and the $C_{i j, r s}$ are integers that depend on the order, but not on $t, u$ or the field $K$. We also have from $[3$, p. 36$]$ :

3.2. For each positive root $r$ there is a homomorphism $\varphi_{r}$ of $S L(2, K)$ into $G$ such that $\varphi_{r}\left(\begin{array}{ll}1 & t \\ 0 & 1\end{array}\right)=x_{r}(t)$ and $\varphi_{r}\left(\begin{array}{ll}1 & 0 \\ t & 1\end{array}\right)=x_{-r}(t)$.

Together with $G$, we consider a covering group $\Gamma$, the abstract group generated by a set of elements $x_{r}(t)\left(t \in K, r \in \sum\right)$ subject to the relations 3.1 and those implied by 3.2 with $\Gamma$ in place of $G$. That these relations define $S L(l+1, K)$ and $S p(l, K)$ for $\Sigma$ of type $A_{l}$ and $C_{l}$ respectively was already known to Dickson [7]. The properities of $\Gamma$ that we require, 3.3 to 3.6 below, are taken from [21].

3.3. Each $\varphi_{r}$ is an isomorphism.

3.4. $\Gamma$ is equal to its commutator subgroup. 
3.5. If $h_{a}(t)=\varphi_{a}\left(\operatorname{diag}\left(t, t^{-1}\right)\right)$ and $h_{a}=\left\{h_{a}(t) \mid t \in K^{*}\right\}$, the $h_{a}(a \in S)$ generate a subgroup $H$ as a direct product.

For each $r \in \sum$ the symbol $r$ is also used to denote the root on $H$ : $\Pi h_{a}\left(t_{a}\right) \rightarrow \Pi t_{a}^{c_{r a}}($ see 2.4).

3.6. The center $C$ of $\Gamma$ consists of those $h \in H$ for which all $r(h)$ are $1 ; C$ is the kernel of the natural projection of $\Gamma$ on $G ; \Gamma / C$ is isomorphic to $G$.

Thus $\Gamma$ acts naturally on $G$-modules, in particular on 8 . Let $\Gamma^{\prime}(K), G(K)$, etc. denote the dependence of $\Gamma, G$ etc. on $K$.

3.7. Let $K$ be algebraically closed and of infinite degree of transcendence over the prime field. Then $G$ and $\Gamma$ may be identified, via isomorphisms, with a simple algebraic group and its simply connected covering group, and both may be defined over the prime field. It is then true that (a) the pth power automorphism of $\Gamma$ is given by $x_{r}(t) \rightarrow x_{r}\left(t^{p}\right)$, (b) if $k$ is a subfield of $K . \Gamma_{k}$ is naturally isomorphic to $\Gamma(k)$, and (c) $H$ is a Cartan subgroup of $\Gamma$.

These results, which cover all simple algebraic groups because of the classification in [13], are proved at the end of $\$ 4$.

We use $\omega_{a}(a \in S)$ to denote the function (fundamental weight) on $H$ defined by $\Pi h_{b}\left(t_{b}\right) \rightarrow t_{a}$, and set $\omega=\Pi \omega_{a}$.

$$
\text { 3.8. } \quad \omega^{2}=\Pi_{r>0} r .
$$

For a proof of the additive version of this result see [14, p. 19-01].

Finally to close this section we prove a result of fundamental importance in our later discussion of the representations of finite groups. We are indebted to T. A. Springer for the main ideas of the proof.

3.9 Lemma. Assume that $K$ is algebraically closed and of infinite transcendence degree over its prime field $F_{p}$, that $\tau$ is a rational automorphism of $\Gamma$ such that $H^{\tau}=H$, that $\sigma$ is the composition of $\tau$ with the pth power automorphism, and that $\Gamma_{\sigma}$ is the subgroup of fixed points of $\sigma$. Then (a) the semisimple classes of conjugate elements of $\Gamma_{\sigma}$ are in natural one-one correspondence with those orbits of $H$ under $W$, the Weyl group, that are invariant under $\sigma$, and (b) if for each $a \in S, \gamma_{a}$ is the sum of the distinct images of $\omega_{a}$ under $W$, the orbit space $H / W$ is an affine variety with coordinates $\gamma_{a}(a \in S)$. 
The proof proceeds in several steps.

(1) Assume that $B$ is a (connected) algebraic subgroup of $\Gamma$ and that $B^{\sigma}=B$. Then for each $x$ in $B$ there is $a y$ in $B$ such that $x=y^{-1} y^{5}$. This result is quite close to one of Lang [9], and it does not depend on the simple-connectedness or semisimplicity of $\Gamma$. The proof, a straightforward modification of Lang's, is omitted.

(2) The centralizer of a semisimple element of a simply-connected semisimple algebraic group is connected. Here are the main steps in a proof due to Springer (unpublished). The semisimple element $h$ is put in a Cartan subgroup $H$, and then by the Bruhat decomposition [13, p. 13-11], the problem is reduced to showing that an element of the Weyl group that leaves $h$ fixed is a product of reflections (corresponding to roots) that also do. After the problem is shifted from $H$ to an ordinary torus $T$ and then to the covering space of $T$, the proof is completed by geometric means.

(3) Two semisimple elements of $\Gamma_{\sigma}$ which are conjugate in $\Gamma$ are also conjugate in $\Gamma_{\sigma}$. Assume $x=z w z^{-1}\left(x, w \in \Gamma_{\sigma}, z \in \Gamma\right)$. Then $x=z^{\sigma} w z^{-\sigma}$, whence $z^{-1} z^{\sigma}$ is in $B$, the centralizer of $w$. By 3.7 and (2), $B$ is connected if $w$ is semisimple, and because $w \in \Gamma_{\sigma}, B^{\sigma}=B$. Thus by (1) we can write $z^{-1} z^{\sigma}=y^{-1} y^{\sigma}$ $(y \in B)$. Then $z y^{-1} \in \Gamma_{\sigma}$, and since $x=\left(z y^{-1}\right) w\left(z y^{-1}\right)^{-1}$, we have (3).

(4) An element of $\Gamma$ is conjugate to an element of $\Gamma_{\sigma}$ if and only if it is conjugate to its image under $\sigma$. For if $z \in \Gamma$, then $z=x z^{\sigma} x^{-1}$ for some $x \in \Gamma$ if and only if $z=y^{-1} y^{s} z^{s} y^{-\sigma} y$ for some $y \in \Gamma$, by (1) with $B=\Gamma$, that is, if and only if $y z y^{-1} \in \Gamma_{\text {o }}$ for some $y \in \Gamma$.

(5) Two elements of $H$ are conjugate in $\Gamma$ if and only if they are conjugate under $W$. This easily comes from the uniqueness in the Bruhat decomposition.

Since an element of $\Gamma$ is semisimple if and only if it is conjugate to an element of $H$ [13, p. 6-13], we may combine (3), (4) and (5) to get (a). In $[15$, p. $57-8]$ it is proved that $H / W$ is an affine algebraic variety whose coordinate ring is got from that of $H$ by selecting the invariants under $W$. This means the polynomials in $\omega_{a}, \omega_{a}^{-1}(a \in S)$ that are symmetric relative to $W$. Thus to complete the proof of (b) we need only establish the following result, which in case $W$ is of type $A_{l}$ reduces to the fundamental theorem for sym. 
metric functions.

(6) Every polynomial in $\omega_{a}, \omega_{a}^{-1}(a \in S)$ that is symmetric relative to $W$ is a polynomial in the elementary symmetric polynomials $\gamma_{a}(a \in S)$. Partially order the monomials $\Pi \omega_{a}^{n_{b}}$ so that each is higher than those obtained by multiplying it by a product of negative (multiplicative) roots. Thus if $\beta$ is a nonzero symmetric polynomial and $c \prod \omega_{a}^{n_{a}}$ is one of its highest terms, each $n_{a} \geq 0$ because $\beta$ is symmetric. Then $\beta-c \Pi \gamma_{a}^{n a}$ does not contain this term, and the proof of (6) may be completed by induction.

\section{§ 4. Lifting representations from algebras to groups}

The notations $p, K, \Sigma, S, H, \omega_{a}$, etc. introduced in $\$ \S 2$ and 3 in connection with the algebra $g$ and corresponding groups $G$ and $\Gamma$ will be used throughout the paper. By a module (or representation) for these groups we mean one over $\bar{K}$, the algebraic closure of $K$. Following Curtis [4], we first convert each $M \in \mathfrak{M}$ into a projective $\Gamma$-module. For $x \in \Gamma$, let $M^{x}$ be the irreducible $g$-module obtained by defining the action of $g$ on $M$ by the rule:

$$
\left(M^{x}\right) \quad X . v=X^{x} v \quad(X \in \mathrm{g}, v \in M) .
$$

Here $X^{x}$ is the image of $X$ under $x$ and we use the convention $\left(X^{x}\right)^{y}=X^{y x}$. The module $M^{x}$ is equivalent to $M$ [4]. Thus there is a $g$-module isomorphism $T(x)$, uniquely determined to within a scalar multiple by Schur's lemma, of $M$ on $M^{x}$. This satisfies:

$$
T(x) X v=X^{x} T(x) v \quad(x \in \Gamma, X \in \mathfrak{g}, v \in M) .
$$

The map $x \rightarrow T(x)$ is a projective representation of $\Gamma$ (or $G$ ) on $M$, again by Schur's lemma. For each positive root $r$ we may (and do) normalize all $T\left(x_{r}(t)\right)$ to keep $v_{+}$fixed (see 2.7); since 4.1 and 4.2 imply that $T\left(x_{r}(t)\right) v=$ $v+$ higher terms, for each monomial $v$, this amounts to making each $T\left(x_{r}(t)\right)$ unipotent. After treating negative roots in a similar way, we want to show that the normalization can be extended to yield an ordinary (not just a projective) representation of $\Gamma$. When it is convenient, we write $x v$ for $T(x) v$.

4.3 Lemma. Let $M \in \mathfrak{M}$ have high weight $\lambda(a)(a \in S)$, fix $a \in S$ and set $\lambda(a)=n$. Then (a) $v_{+}, X_{-a} v_{+}, \ldots, X_{-a}^{n} v_{+}$are linearly independent and $X_{-a}^{n+1} v_{+}=0$; (b) the normalized action of the $x_{a}(t)$ and $x_{-a}(t)$ on $M$ can be 
extended to an ordinary representation of $Z_{a}$, the group generated by these elements; we then have (c) $h_{a}(t) v_{+}=t^{n} v_{+}$.

Proof. By induction, $X_{a} X_{-a}^{i} v_{+}=i(n-i+1) X_{-a}^{i-1} v_{+}(i \geq 1)$, whence the vectors $X_{-a}^{i} v_{+}(0 \leq i \leq n)$ are nonzero and then linearly independent by 2.9. Further $X_{a} X_{-a}^{n+1} v_{+}=0$, and clearly $X_{r} X_{-a}^{n+1} v_{+}=0$ for $r>0, r \neq a$. Thus $X_{-a}^{n+1} v_{+}=0$ by 2.7 and 2.9. Now set $v_{0}=v_{+}, i v_{i}=X_{-a} v_{i-1}(1 \leq i \leq n)$, so that also $(n-i) v_{i}$ $=X_{a} v_{i+1}$. From $x_{a}(t) v_{+}=v_{+}, x_{a}(t) X_{-a}=X_{-a}+t H_{a}-t^{2} X_{a}$ and 4.2 , we see by induction that $x_{a}(t) v_{i}=\sum_{j=0}^{i}\left(\begin{array}{c}n-j \\ n-i\end{array}\right) t^{i-j} v_{j}$. Now interchanging the roles of $X_{a}$ and $X_{-a}$, and also of $v_{0}$ and $v_{n}$, that is, replacing $M$ by $M^{w}$ with $w$ an element of $Z_{a}$ corresponding to the Weyl reflection relative to $a$, we get $x_{-a}(t) v_{i}$ $=\sum_{j=i}^{n}\left(\begin{array}{c}j \\ i\end{array}\right) t^{j-i} v_{j}$. Introducing a space with coordinates $x$ and $y$ and setting $v_{i}^{\prime}=x^{n-i} y^{i}$, we see that in the space of polynomials of degree $n$ exactly the same equations hold for the transformations $x_{a}^{\prime}(t): x, y \rightarrow x, y+t x$ and $x_{-a}^{\prime}(t)$ : $x, y \rightarrow x+t y, y$. We thus see that the relations on the $x_{a}(t)$ and $x_{-a}(t)(a \in S)$ implied by 3.2 also hold for the $T\left(x_{a}(t)\right)$ and $T\left(x_{-a}(t)\right)$. Further the relations 3.1 with $r, s>0$ are also preserved, as we see by applying both sides to $v_{+}$ and noting that every term leaves $v_{+}$fixed. Since we may choose an element $w$ in $\Gamma$ corresponding to an arbitrary' element of the Weyl group and apply the above considerations to $M^{w}$, we see that all of the relations of 3.1 and 3.2 are preserved. We thus get $4.3(\mathrm{~b}), 4.3(\mathrm{c})$ and also:

4.4. The projective representation of $I$ ' on $M$ can be lifted in a unique way to an ordinary representation.

The uniqueness comes from 3.4, which implies that $\Gamma$ has no nontrivial one-dimensional representation.

From the definitions we see that if $v$ has height $n$ in $M$ then

$$
\left(x_{r}(t)-1\right) v=t X_{r} v+\text { higher (lower) terms, when } r>0 \quad(r<0) .
$$

By 2.7 this yields :

4.6. The vectors $\bar{K} v_{+}$are the only ones fixed by all $x_{r}(t)(r>0)$.

From this and 4.3, we see that the $\Gamma$-module $M$ determines $\bar{K} v_{+}$, which in turn determines $\lambda(a)(a \in S)$ since $\lambda(a)+1$ is the dimension of the subspace generated by the elements $x_{-a}(t)(t \in K)$ acting on $\bar{K} v_{+}$. Thus using also $4.3(\mathrm{c})$, 
4.7. If $M_{1}$ and $M_{2}$ in $\mathfrak{M}$ are distinct as $\mathrm{g}$-modules, they are distinct as $\Gamma$-modules. If $M_{1}$ has high weight $\lambda$ as $\mathrm{g}$-module, it has high weight $\Pi \omega_{a}^{\lambda(a)}$ as $\Gamma$-module.

In order to pass to projective $G$-modules, we use :

4.8. Under the natural projection from $\Gamma$ to $G$, each irreducible $\Gamma$-module leads to an irreducible projective G-module. Distinct $\Gamma$-modules yield distinct G-modules.

We need only observe that the center $C$ of $\Gamma$ must act via scalars in any irreducible representation of $\Gamma$, and then use 3.4.

Now $\Gamma$ acts faithfully on $\mathfrak{M}$ as a set: since $\Gamma / C$ is simple, the kernel is contained in $C$ and consists of those $h$ for which all $\omega_{a}(h)(a \in S)$ are 1 . Further, relative to monomial bases, the group $\left\{x_{r}(t), t \in K\right\}$ acts via matrices that are polynomials in $t$ with coefficients in $F_{p}$, thus acts as an algebraic group defined over $F_{p}$ with the $p$ th power map given by $x_{r}(t) \rightarrow x_{r}\left(t^{p}\right)$. The same is thus true of the group $\Gamma$. Comparing the structure just put on $\Gamma$ with the one put on $G$ by $T$. Ono [J. Math. Soc. Japan 10 (1958)], and using his results and methods and those of [13, Exp. 23], we easily get the assertions of 3.7 and also

4.9. $\Gamma$ (hence also $G$ ) acts rationally on each $M$ in $\mathfrak{M}$.

\section{$\S 5$. Tensor product theorem}

Each isomorphism $\alpha$ of $K$ into $\bar{K}$ gives rise to an isomorphism of $\Gamma(K)$ into $I(\bar{K})$, defined by $x_{r}(t) \rightarrow x_{r}\left(t^{\alpha}\right)$, and can thus be used to convert each $\Gamma$ module $M \in \mathfrak{M}$ into another $\Gamma$-module, denoted $M^{\alpha}$, by the rule $x \cdot v=x^{\alpha} v$ $(x \in \Gamma(K), v \in M)$.

5.1 Theorem. (a) If $M_{1}, M_{2}, \ldots, M_{k}$ are in $\mathfrak{M}$ and $\alpha_{1}, \alpha_{2}, \ldots, \alpha_{k}$ are distinct isomorphisms of $K$ into $\bar{K}$, then $M=M_{1}^{\alpha_{1}} M_{2}^{\alpha_{2}} \cdots M_{k}^{\alpha_{k}}$ (tensor product) is an irreducible $\Gamma$-module. (b) For a fixed sequence of $\alpha$ 's, two $\Gamma$-modules $M$ constructed in this way are equivalent if and only if the sequences of $M_{i}^{\prime}$ s are the same. Or, equivalently, if $N=N_{1}^{\beta_{1}} N_{2}^{\beta_{2}} \cdots N_{l}^{\beta_{l}}$ with the $N_{j}$ in $\mathfrak{M}$ and the $\beta_{j}$ distinct isomorphisms of $K$ into $\bar{K}$, then $M$ is equivalent to $N$ if and only if, after the deletion of all one-dimensional factors, $k=l$ and, for some permutation $\pi$ of $1,2, \ldots, k, M_{i}$ is equivalent to $N_{\pi i}$ and $\alpha_{i}=\beta_{\pi i}$ for $i=1,2, \ldots, k$. 
(c) If the modules in (a) and (b) are taken to be projective G-modules, the modified statements are also true.

Proof. Let $X_{r}^{(i)}$ be the transformation that is $X_{r}$ on $M_{i}^{\alpha_{i}}$ and the identity on the other components of $M$, let $v_{+}=\Pi v_{+}^{(i)}$ be the product of the high vectors in the separate components, and let the height of a product of monomials be defined as the sum of the heights of its terms.

(1) The set of vectors of $M$ annihilated by all $X_{r}^{(i)}(r>0, i=1,2, \ldots, k)$ is $\bar{K} v_{+}$. Nonzero vectors of distinct heights are linearly independent. For $k=1$, this follows from 2.7 and 2.9. If $k>1$, we can write any $v$ in $M$ as $v=\sum u_{j} w_{j}$ $\left(u_{j} \in M_{1}^{\alpha_{1}} \cdots M_{k-1}^{\alpha_{k-1}}, w_{j} \in M_{k}^{\alpha_{k}}\right)$ with the $u_{j}$ and also the $w_{j}$ linearly independent. Then $X_{r}^{(k)} v=\sum u_{j}\left(X_{r} w_{j}\right)$, which is 0 only if all $X_{r} w_{j}$ are 0 , because the $u_{j}$ are linearly independent. Thus $X_{r}^{(k)} v=0$ for all $r>0$ only if all $w_{j}$ are in $\bar{K} v_{+}^{(k)}$, whence the first part of (1) follows by induction. This implies the second part (see the proof of 2.9) and also:

(2) If $v$ is a homogeneous vector of $M$ and $r>0$ (resp. $r<0)$, then $\left(x_{r}(t)-1\right) v=\sum t^{\alpha_{i}} X_{r}^{(i)} v+$ higher (resp. lower) terms.

(3) Irreducibility. Let $M^{\prime}$ be a $\Gamma$-submodule of $M$ and $v$ a nonzero vector of $M^{\prime}$. Write $v=v_{0}+v_{1}+\cdots+v_{d}, v_{d} \neq 0$, height $v_{j}=-j$. The $\alpha_{i}$ are distinct, thus linearly independent. By (2) this implies that for every $r>0$ and every $i=1,2, \ldots, k$ there is a vector $X_{r}^{(i)} v_{d}+$ higher terms in $M^{\prime}$, whence by (1) and induction on $d$ the vector $v_{+}$is also in $M^{\prime}$. Then using negative roots, we see by (downward) induction on the height that for every monomial $v$ of $M$ there is a vector $v$ +lower terms in $M^{\prime}$. By induction on the height this implies that $M^{\prime}$ contains all monomials, that $M^{\prime}=M$, that $M$ is irreducible.

(4) Uniqueness. Let $\lambda_{i}$ be the high weight of $M_{i}$ as $g$-module. We must show that $M$ as $\Gamma$-module intrinsically determines the numbers $\lambda_{i}(a)(i=1,2$, $\ldots, k ; a \in S)$. First note that $M$ determines $\bar{K} v_{+}$by (1). Fix $a$ and set $\lambda_{i}(a)=a_{i}$. If all $x_{-a}(t)(t \in K)$ fix $v_{+}$, then the $a_{i}$ are certainly determined by $M$-they must all be 0 by 4.3 (a) and 4.5. Assume henceforth that this is not the case. Then $h_{a}(t)$ acts on $\bar{K} v_{+}$with the characteristic value $t^{\alpha}, \alpha=\sum a_{i} \alpha_{i}$, some $a_{i} \neq 0$, by 4.3 (c). Assuming that $M$ does not determine the $a_{i}$ uniquely, we are thus led to the existence of a nontrivial identical relation $t^{\alpha}=t^{\beta}$ ( $\beta=$ $\sum b_{i} \alpha_{i}$, some $b_{i} \neq 0$, some $\left.b_{j} \neq a_{j}, t \in K\right)$. Among all such relations on the 
monomials $t^{\gamma}\left(\gamma=\sum c_{i} \alpha_{i}, 0 \leq c_{i} \leq p-1\right)$, ordered lexicographically, we choose one of minimum degree. The substitution $t \rightarrow t u$ shows that this relation has the form $t^{\delta}=t^{\varepsilon}\left(\delta>\varepsilon, \delta=\sum d_{i} \alpha_{i}, \varepsilon=\sum e_{i} \alpha_{i}\right)$. Then using the minimality of the degree and the substitution $t \rightarrow t+u$, we get in turn $d_{k}=0$ (whence we may assume $e_{k} \neq 0$ since otherwise the proof is completed by induction), $k>1$, $\delta=\alpha_{1}, \varepsilon=\alpha_{k}$, and $\alpha_{1}=\alpha_{k}$, a contradiction. This proves the first statement of (b). The second follows immediately.

(5) G-modules. By 4.8 the results we have proved for $T$-modules are equally valid for $G$-modules, which is (c).

We remark that considering $g$ as Lie ring rather than Lie algebra we can interpret $M_{i}^{\alpha}{ }_{i}$ above as $g$-module and then prove a theorem entirely analogous to 5.1 with $\mathfrak{g}$ in place of $\Gamma$.

\section{$\S 6$. Rational representations}

As a first application of 5.1 we have:

6.1 THEOREM. If $K$ is infinite and perfect and $\alpha_{i}$ denotes the automorphism $t \rightarrow t^{p^{i}}$ of $K$, then every irreducible rational $\Gamma$-module or irreducible rational projective G-module can be expressed uniquely as a tensor product $M=\Pi_{i=0}^{\infty} M_{i}^{\alpha_{i}}$ $\left(M_{i} \in \mathfrak{M}\right.$, almost all $M_{i}$ trivial).

Proof. By 4.8 we need only consider $\Gamma$-modules, and by the density theorem of Rosenlicht [12, p. 44] we may assume that $K$ is algebraically closed and of infinite transcendence degree over its prime field. For given, but arbitrary, nonnegative integers $\lambda(a)(a \in S)$, we can uniquely write $\lambda(a)=$ $\sum p^{i} \lambda_{i}(a)\left(0 \leq \lambda_{i}(a) \leq p-1\right)$, choose $M_{i}$ in $\mathfrak{M}$ as the $I^{\prime}$-module with high weight $\Pi \omega_{a}^{\lambda_{i}(a)}$ (see 4.7 ), and thus construct a $\Gamma$-module $\Pi M_{i}^{\alpha_{i}}$ which is rational by 4.9, irreducible by 5.1 , and has high weight $\Pi \omega_{a}^{\lambda(a)}$. Using the classification [13, Exp. 14 and 15] of irreducible representations of semisimple algebraic groups in terms of high weights, we see that this construction yields a complete set of irreducible rational $\Gamma$-modules, whence 6.1 .

By now we have also shown that if $K$ is infinite and perfect every irreducible rational projective representation of $\Gamma$ or $G$ comes in a unique way from an

ordinary representation of $\Gamma$. 


\section{$\S 7$. Finite groups, normal forms}

If $K$ is finite with $q$ elements, we write $\Gamma_{q}, G_{q}$ for $\Gamma, G$. The following fact, not used here, is proved in [21].

7.1. If the rank is at least 2 , the relations 3.1 alone are enough to define $I_{q}$.

What is required here from [21] is:

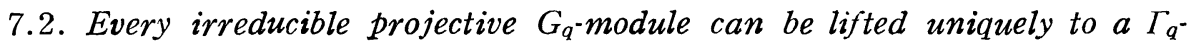
module.

Now by 3.9 with $\sigma$ the $q$ th power mapping, the semisimple classes of conjugate elements of $I_{q}$ are characterized by coordinates $\gamma(a)$ subject to the condition $\gamma(a)^{q}=r(a)$ (see also 3.7(a)). This yields:

7.3 LEMMA. If the rank is $l$, the number of semisimple classes of conjugate elements of $I_{q}$ is $q^{l}$.

We can now prove one of the main results of this paper. Observe that the modules considered are not assumed to be rational, throughout this section.

7.4 Theorem. Let $q=p^{n}$ and let $\alpha_{i}$ denote the field automorphism $t \rightarrow t^{p^{i}}$. Then every irreducible $\Gamma_{q}$-module, also every irreducible projective $G_{q}$-module, can be expressed uniquely as a tensor product $M=\prod_{i=0}^{n-1} M_{i}^{\alpha_{i}}\left(M_{i} \in \mathfrak{M}\right)$. If the rank of $\Gamma_{q}$ is $l$, there are $q^{l}$ such modules.

Proof. Again we need only consider $\Gamma_{q}$-modules, this time by 7.2 . By 7.3 and a theorem of Brauer and Nesbitt [1, p. 14] the number of inequivalent irreducible $\Gamma_{q}$-modules is $q^{l}$. Since the $q^{l}$ modules $\Pi M_{i}^{\alpha_{i}}$ are inequivalent and irreducible by 5.1 , they thus form a complete set, as required.

7.5 Corollary. If $L$ is an infinite field containing the finite field $K$, every irreducible representation of $I^{\prime}(K)$ can be extended to $\Gamma(L)$. Every irreducible representation of $\Gamma(K)$ can be realized over $K$. The corresponding statements for projective representations of $G$ are also true.

The first statement is clear. For the second we need only observe that relative to a monomial basis 2.8 for $M_{i} \in \mathfrak{M}$ each generator $x_{r}(t)$ is represented by a matrix which is a polynomial in $t$ with coefficients in the prime field.

The modules of 7.4 have high weights $\Pi \omega_{a}^{\lambda(a)}$ with $0 \leq \lambda(a) \leq q-1$. Those in which the center $C$ of $\Gamma$ (see 3.6) acts trivially, or what is equivalent, fixes 
$v_{+}$, yield all of the irreducible $G_{q}$-modules. Thus

7.6 Corollary. Every irreducible $G_{q}$-module is obtained from a $\Gamma_{q}$-module for which the high weight $\Pi \omega_{a}^{\lambda(a)}$ is 1 on the center of $\Gamma$.

In individual cases, when the root system $\Sigma$ is specfied, more detailed results can be given. Thus if $\sum$ is of type $E_{8}, F_{4}$ or $G_{2}$ (and $K$ is arbitrary), $\Gamma=G$, so that 7.6 is superfluous, while, for example, if $\sum$ is of type $A_{l}$ (so that $\Gamma_{q}$ and $G_{q}$ are respectively isomorphic to $\operatorname{SL}(l+1, q)$ and $\operatorname{PSL}(l+1, q)$, the irreducible $G_{q}$-modules correspond to the sequences $\left(\lambda_{1}, \lambda, \ldots, \lambda_{l}\right)$ for which $0 \leq \lambda_{i} \leq q-1$ and $\sum i \lambda_{i}$ is divisible by the greates common divisor of $l+1$ and $q-1$.

Dually, one can make similar statements concerning the classes of conjugate elements of $G$ in terms of those of $\Gamma$.

Finally, we remark that there are results analogous to 7.4 and 7.5 with the finite Lie ring $g_{q}$ in place of $\Gamma_{q}$. The proof of completenteness of the modified 7.4 can be given along the lines of $[14 ;$ p. 22-11, 22-12].

\section{$\S 8$. Prime power representations}

A general formula in characteristic $p$, comparable to Weyl's formula in characteristic 0 (cf. [6]), for the characters or dimensions of the above modules does not yet exist (except for groups of type $A_{1}$ and $A_{2}[13$, p. 588], [14]). However, for the irreducible $\Gamma_{q}$-module with the greatest of all possible high weights, $\omega^{q-1}$ (recall that $\omega=\Pi \omega_{a}$ ), that is, the module $M_{q}=\Pi M_{i}^{\alpha_{i}}$ of 7.4 in which each $M_{i}$ is equivalent to the module $M_{p}$ of $\mathfrak{D}$ ith wigh weight $\lambda(a)=p-1$ $(a \in S)$ as $g$-module, the situation can be described rather completely and is very much as in characteristic 0 . The following result is proved in [17].

8.1 Lемм. If $m$ is the number of positive roots in $\sum$, there is an irreducible $\Gamma_{q}$-module $\bar{M}_{q}$ of dimension $q^{m}$, that is, the order of a p-Sylow subgroup of $\Gamma_{q}$.

8.2 Theorem. The $\Gamma_{q}$-modules $\bar{M}_{q}$ of 8.1 and $M_{q}$ of high weight $\omega^{q-1}$ are eqnivalent. All other irreducible $\Gamma_{q}$-modules have smaller dimensions than $M_{q}$.

Proof. Because each module of $\mathfrak{M}$ is spanned by the monomials 2.8 , the only one that could have a dimension as large as $p^{m}$ is $M_{p}$ by $4.3(\mathrm{a})$, and hence by 7.4 the only possible irreducible $\Gamma$-module of dimension as large as 
$q^{m}$ is $M_{q}$. From the existence of the module $\bar{M}_{q}$ with dimension $q^{m}$, it follows that $M_{q}$ is equivalent to $\bar{M}_{q}$ and that the other irreducible $\Gamma_{q}$-modules have smaller dimensions.

In the course of the argument, we have proved:

8.3 Corollary. The irreducible module $M_{p}$ with high weight $\omega^{p-1}$ has dimension $p^{m}$ and a basis consisting of all the monomials 2.8.

A direct proof of this result, within the framework of g-modules, also exists. Using 8.3 we can compute the (Brauer) character of $M_{q}$. To define this we write the order $g$ of $\Gamma_{q}$ as $g=p^{e} g^{\prime},\left(p, g^{\prime}\right)=1$, choose an isomorphism $\theta$ of the group of $g^{\prime}$ th roots of 1 in $\bar{K}$ onto the corresponding group in the complex field, and then for any semisimple element $x$ of $\Gamma_{q}$ and any module $M$ for $\Gamma_{q}$, define $\chi(x)$, the character of $x$ on $M$, to be $\sum \theta\left(c_{i}\right)$, the sum to be taken over the characteristic roots $c_{i}$ of $x$ on $M$. Generally $\chi$ depends on the choice of $\theta$, but not on $M_{q}$ where it turns out to be rational.

The following result has been proved previously only for groups of type $A_{l}[16$, p. 281$]$ and in somewhat different terms.

8.4 TheOREM. If $\Gamma_{q}$ is of rank $l$, and $x$ is a semisimple element whose centralizer in the correpsonding algebraic group has dimension $l+2 d(x)$, or equivalently, whose action on $\mathfrak{g}$ has fixed point set of dimension $l+2 d(x)$, the character of $x$ on $M_{q}$ is given by $\chi(x)= \pm q^{d(x)}$.

Proof. In $\Gamma(\bar{K}), x$ is conjugate to an $h$ in $H(\bar{K})$. Now since $h$ acts on the monomial 2.8 of $M_{p}$ by multiplication by $\omega^{p-1}(h) \Pi r_{k}(h)^{-i_{k}}$, we see by 8.3 that the character of $h$ on $M_{p}$ is

$$
\psi(h)=\theta\left(\omega^{p-1}(h)\right) \Pi_{r>0} \sum_{i=0}^{p-1} \theta\left(r(h)^{-i}\right),
$$

and then using 3.8 , that the character $\chi(h)$ on $M_{q}$ satisfies $\chi(h)^{2}=\Pi_{r} \sum_{i=0}^{q-1}$ $\theta\left(r(h)^{(q-1-2 i) / 2}\right)$, the product over all roots. Since $h$ is conjugate to an element of $I_{q}$ and the roots are permuted by the Weyl group, it follows from (4) and (5) of the proof of 3.9 that the numbers $\theta(r(h))^{q}$ form a permutation of the numbers $\theta(\boldsymbol{r}(h))$. Thus the roots can be arranged in cycles (of various lengths) $\left(r_{1} r_{2} \cdots r_{k}\right)$ such that $\theta\left(r_{i}(h)\right)^{q}=\theta\left(r_{i+1}(h)\right), \theta\left(r_{k}(h)\right)^{q}=\theta\left(r_{1}(h)\right) . \quad$ If $r(h) \neq 1$, the cycle containing $r$ telescopically contributes 1 to the product for $\chi(h)^{2}$, since the term for $r$ may be written, subject to a consistent choice of square roots, 
as $\left(c^{q / 2}-c^{-q / 2}\right) /\left(c^{1 / 2}-c^{-1 / 2}\right)$ with $c=\theta(r(h))$. The terms for which $r(h)=1$ contribute $q$ each to the product, $q^{2 d(h)}$ together. Thus $\chi(h)^{2}=q^{2 d(h)}$. Since $h$ is conjugate to $x$, we have 8.4 .

8.6 Corollary. If $\Gamma_{q}$ is replaced by $G_{q}, 8.2$ and 8.4 remain valid.

We need only remark that $M_{p}$, hence $M_{q}$, is an ordinary (not just projective) $G_{q}$-module: if $c$ is in the center of $\Gamma, \omega^{2}(c)=\Pi r(c)=1$ by 3.6 and 3.8 , whence $\omega^{p-1}(c)=1$, even if $p$ is 2 .

Finally we remark that for semisimple algebraic groups over an algebraically closed field of characteristic 0 , results analogous to 8.3 to 8.6 , in which $p$ need not be a prime nor $q$ a prime power are true. Here we content ourselves with showing that the formula 8.5 for the character on the irreducible module with high weight $\omega^{p-1}$ is essentially unchanged. With $\Delta(j)=\sum(\operatorname{det} w)(w \omega)^{j}$, the sum over the Weyl group, Weyl's formula for the character [26, p. 389] yields $\Delta(p) / \Delta(1)$, that is, 8.5 with $\theta=1$ because of the basic factorization $\Delta(j)=\omega^{j} \Pi_{r>0}\left(1-r^{-j}\right)[26$, p. 386$]$.

\section{§. Finite groups, nonnormal forms}

In this section we treat the simple groups denoted as $A_{l}^{1}$ (a projective unitary group in $l+1$ dimensions), $D_{l}^{1}$ (a second projective orthogonal group in $2 l$ dimensions), $E_{6}^{1}$ (a nonnormal "real" form of $E_{6}$ ) and $D_{4}^{2}$ (a "triality" form of $D_{4}$ ) in [19], and their covering groups. Each of the latter groups can be defined in terms of generators and relations derived from the structure of the corresponding simple group, just as $\Gamma$ is in terms of $G$ in $\S 3$; however, it is more convenient to define them directly as subgroups of the groups $\Gamma$. Starting with an automorphism $\sigma$, other than the identity, of the root system $\sum$ such that $\sigma S=S$, and an automorphism $\sigma$ of the same period on the field $K$, we can construct an automorphism, also called $\sigma$, of the corresponding group $\Gamma$ such that $x_{a}(t)^{\sigma}=x_{\sigma a}\left(t^{\sigma}\right)$ for all $a \in \pm S$ and all $t \in K$, and then define $\Gamma^{1}$ to be the group of fixed points of $\sigma$. Comparing this definition with the one given in [19] for the corresponding simple groups, and using 3.6, we easily get:

9.1. Let $C^{1}$ be the center of $\Gamma^{1}$. Then $C^{1}=C \cap \Gamma^{1}$, and $\Gamma^{1} / C^{1}$ is naturally isomorphic to the corresponding simple group of [19].

We write $G^{1}$ for $\Gamma^{1} / C^{1}, K_{0}$ for the fixed field under $\sigma$, and $\Gamma_{q}^{1}$, etc. when $K_{0}$ 
has $q$ elements. As a subgroup of $T, \Gamma^{1}$ acts naturally on each $\Gamma$-module.

9.2 Theorem. If the $M_{i}$ are in $\mathfrak{M}$, and the $\alpha_{i}$ are isomorphisms of $K$ into $\bar{K}$ which are distinct on $K_{0}$, then $M=M_{1}^{\alpha_{1}} M_{2}^{\alpha_{2}} \cdots M_{k}^{\alpha_{k}}$ is an irreducible $\Gamma^{1}$ module, and there is uniqueness in this product representation in the sense of the second sentence of $5.1(\mathrm{~b})$.

Proof. We use the notations of the proof of 5.1 and assume that $\sigma$ above has period 2. If the period is 3 , as it is for one of the groups of type $D_{4}$, the argument is similar. If $r$ is a root such that $o r=r$ and there is no root $s$ such that $r=s+\sigma s$, then we may assume $x_{r}(t) \in \Gamma^{1}$ for all $t \in K_{0}[19, p$. 879]; and we have 5.1(2) holding. If $r$ is such that $o r \neq r$ and $r+o r$ is not a root, then (see [19]) $x_{r}(t) x_{r r}\left(t^{\sigma}\right) \in \Gamma^{1}$ for all $t$ in $K$, and we have instead $\left(x_{i}(t) x_{\sigma r}\left(t^{\sigma}\right)-1\right) v=\sum t^{\alpha_{i}} X_{r}^{(i)} v+\sum t^{\sigma \alpha_{i}} X_{\sigma r}^{(i)} v+\cdots$. If $r$, or and $r+\sigma r$ are all roots, the situation is similar. Since the $\alpha_{i}$ act distinctly on $K_{0}$ and the $\alpha_{i}$ and $\sigma \alpha_{i}$ together act distinctly on $K$, the proofs of irreducibility and uniqueness in 9.2 are from this point on straightforward modifications of those in 5.1.

For finite groups, we have:

9.3 Theorem. If $K_{0}$ has $q=p^{n}$ elements and $\alpha_{i}$ denotes the field automorphism $t \rightarrow t^{p^{i}}$, then every irreducible $I_{q}^{1}$-module can be written uniquely as a tensor product $M=\Pi_{i=0}^{n-1} M_{i}^{\alpha_{i}}\left(M_{i} \in \mathfrak{M}\right)$. If the rank is $l$, the number of such modules is $q^{l}$.

Thus every irreducible $\Gamma_{q}^{1}$-module is the restriction of some $I^{\prime}(\bar{K})$-module, and the largest high weight that occurs is $\omega^{q-1}$. Again, by 9.2, we are reduced to showing that the number of semisimple classes of conjugate elements is $q^{l}$. By 3.9, these are characterized by coordinates $\gamma(a)(a \in S)$ subject to the condition $\gamma(a)^{q}=\gamma(\sigma a)$. Whatever permutation $\sigma$ effects on $S$, the number of solutions is $q^{l}$ (the contribution for each cycle of length $d$ is $q^{d}$ ), as required.

Turning again to [21], we have:

9.4. If the type $A_{l}$ (l even) is excluded, every irreducible projective $G_{q^{-}}^{1}$ module can be lifted uniquely to a $\Gamma_{q}^{1}$-module.

Quite likely this exclusion is unnecessary, but we have not yet shown this. From 9.3 and 9.4 we get:

9.5 Corollary. If the type $A_{l}$ ( $l$ even) is excluded, 9.3 also holds for 
projective $G_{q}^{1}$-modules.

Here also one can get the irreducible $G_{q}^{1}$-modules as those of $\Gamma_{q}^{1}$ in which the center acts trivially. Since 8.1 is true with $\Gamma_{q}^{1}$ in place of $\Gamma_{q}[17,19]$, the same is true of 8.2. Also if $h$ in $H(\bar{K})$ is conjugate to an element of $\Gamma_{q}^{1}$, then by (4.) and (5) of the proof of 3.9, it is conjugate under the Weyl group to $h^{\sigma}$, so that the numbers $r(h)^{q}$ again form a permutation of the numbers $r(h)$ (see the definition of $\sigma$ at the beginning of this section). Thus the proof 8.4. carries over as is.

9.6 Theorem. The statements $81,8.2$ and 8.4 are true with $I_{q}^{1}$ in place of $\Gamma_{q}$.

\section{$\S 10$. Special isogenies, infinitesimal and global}

In this section we present a discussion of the rather special isogenies that exist for simple groups of type $B_{l}, C_{l}$ and $F_{4}$ and characteristic 2, and type $G_{2}$ and characteristic 3 (cf. [23, p. 282], [13, Exp. 21-24]). The results will be used in the next sections, where we return to group representations.

In what follows we identify two root systems that are related by a scalar multiplication. Associated with each system $\sum$, there is a dual system $\Sigma^{*}$ and a map of $\sum$ onto $\sum^{*}$ such that $r^{*}=2 r /(r, r)\left(r \in \sum\right)$. When roots of unequal lengths occur, this map preserves angles, sends short roots to long roots and vice versa, maps the simple set $S$ onto another, and puts types $B_{l}$ and $C_{l}$ in duality with each other and types $C_{2}, F_{4}$ and $G_{2}$ with copies of themselves.

The pair $\left(\sum, p\right)$ will be called special if $\sum$ contains roots $r$ and $s$ such that $(s, s) /(r, r)=p$. The possibilities are those listed in the first paragraph of this section. In the corresponding algebra $g$ of $\S 2$, those $X_{r}$ and $H_{r}$ for which $r$ is short span an ideal, denoted $g_{1}$ in what follows. To see this, observe that if $r$ is short and $s$ is long $c_{s r}=p c_{r s}$, and that if $r, s$ and $r+s$ are roots with $r$ short and $r+s$ long then $s$ is short and $p_{r s}=p$ (check for $\sum$ of type $C_{2}$ and $G_{2}$ ). Observe also that in the present case $I^{\prime}=G, \Gamma$ maps $g_{1}$ onto itself (because each $x_{r}(t)$ does), and, being simple, $\Gamma$ acts faithfully on each of $g_{1}$ and $g / g_{1}$. To indicate the dependence of $g$, etc. on $\sum$ we write $g\left(\sum\right)$, etc.

\section{1 (Existence of isogenies). If $\left(\sum, p\right)$ is special, it is possible to normalize}


equations 2.6 for $g\left(\sum\right)$ and $\mathfrak{g}\left(\Sigma^{*}\right)$ so that the following hold. (a) There exists a homomorphism $\bar{\theta}$ of $\mathrm{g}\left(\sum\right)$ into $\mathrm{g}\left(\Sigma^{*}\right)$ such that $\bar{\theta} X_{r}=X_{r^{*}}$ if $r$ is long, $\bar{\theta} X_{r}$ $=p X_{r^{*}}=0$ if $r$ is short, and similar equations hold for $\bar{\theta} H_{r} . \quad$ (b) The kernel of $\bar{\theta}$ in $(\mathrm{a})$ is $\mathrm{g}_{1}\left(\sum\right)$. Thus $\bar{\theta}$ induces an isomorphism, also dented $\bar{\theta}$, of $\left(\mathrm{g} / \mathrm{g}_{1}\right)\left(\sum\right)$ onto $\mathrm{g}_{1}\left(\Sigma^{*}\right)$. (c) If $\Gamma\left(\sum\right)$ acts on $\left(\mathrm{g} / \mathrm{g}_{1}\right)\left(\sum\right)$ and $\Gamma\left(\Sigma^{*}\right)$ on $\mathrm{g}_{1}\left(\sum^{*}\right)$, the map $\theta: x^{\theta}=\bar{\theta} x \bar{\theta}^{-1}\left(x \in \Gamma\left(\sum\right)\right)$ is an isomorphism of $\Gamma\left(\sum\right)$ into $\Gamma\left(\Sigma^{*}\right)$ such that $x_{r}(t)^{\theta}=x_{r^{*}}(t)$ if $r$ is long, $x_{r}(t)^{\theta}=x_{r^{*}}\left(t^{p}\right)$ if $r$ is short, and similar equations hold for $h_{r}(t)^{0}$.

The proof that we have in mind for $\left(\sum, p\right)$ of type $\left(G_{2}, 3\right)$ involves many details and will not be given here. When $p=2$, however, the situation is quite simple since $-1 \equiv 1(\bmod 2)$, and no normalization is required.

Proof of 10.1 for $p=2$. To show that the equations of (a) define a homomorphism, we must verify that the relations 2.1 to 2.6 are preserved. For this the relations 2.2 and 2.6 will suffice since they together with the relations $\left[H_{r}, X_{r}\right]=2 X_{r}=0$ and $\left[X_{r}, X_{-r}\right]=H_{r}$, which are clearly preserved, imply all of the others (cf. [21]). We give details only for 2.6. Now if either $r$ or $s$ is short, then 2.6 is preserved (both sides go to 0 ) because $g_{1}\left(\sum\right)$ is an ideal, while if $r$ and $s$ are long and linearly independent, then either $(r, s)<0$, whence $p_{r s}=1=p_{r^{*} s^{*}}$, or $(r, s) \geq 0$, whence $p_{r s}=0$ and $p_{r^{*} s^{*}}=0$ or 2 . Since $p=2$, we have (a), and then (b). For the proof of (c), we fix a long root $s \in \Sigma$. If $r$ is long, $r \in \sum$, then either $r=-s$ and $x_{r}(t)^{\theta} X_{s^{*}}=\bar{\theta} x_{r}(t) X_{-r}=\bar{\theta}\left(X_{-r}+t H_{r}-t^{2} X_{r}\right)$ $=X_{-r^{*}}+t H_{r^{*}}-t^{2} X_{r^{*}}=x_{r^{*}}(t) X_{-r^{*}}=x_{r^{*}}(t) X_{s^{*}}, \quad$ or $\quad r \neq-s \quad$ and $\quad x_{r}(t)^{\theta} X_{s^{*}}=$ $\bar{\theta} x_{r}(t) X_{s}=\bar{\theta}\left(X_{s}+p_{r s} t X_{r+s}\right)=X_{s^{*}}+p_{r^{*} s^{*}} t X_{r^{*}+s^{*}}=x_{r^{*}}(t) X_{s^{*}}$; whereas if $r$ is short, $r \in \sum$, then either $r+s \notin \sum$ in which case $r^{*}+s^{*} \notin \sum^{*}$ and $x_{r}(t)^{\theta} X_{s^{*}}=X_{s^{*}}=$ $x_{r^{*}}\left(t^{p}\right) X_{s^{*}}$, or $r+s \in \sum$ in which case $2 r+s \in \sum, \quad(2 r+s)^{*}=r^{*}+s^{*}$ and $x_{r}(t)^{\theta} X_{s^{*}}=\bar{\theta} x_{r}(t) X_{s}=\bar{\theta}\left(X_{s}+t X_{r+s}+t^{2} X_{2 r+s}\right)=X_{s^{*}}+t^{2} X_{r^{*}+s^{*}}=x_{r^{*}}\left(t^{2}\right) X_{s^{*}}$. Since the $X_{s^{*}}$ (s long) generate $g_{1}\left(\Sigma^{*}\right)$, we have $(c)$.

10.2 Corollary (well known). Over a perfect field of characteristic 2 the groups $\Gamma$ of type $B_{l}$ and $C_{l}$ are isomorphic.

\section{$\S 11$. Special algebraic groups}

In case $(\Sigma, p)$ is special our previous results on representations can be refind. In $\mathfrak{M}$ let $\mathfrak{M}^{\prime}\left(\mathfrak{M}^{\prime \prime}\right)$ be the subset each of whose elements has, as 
g-module, high weight $\lambda$ vanishing on all long (short) roots of $S$.

11.1 Theorem. Assume that $(\Sigma, p)$ is special and regard the elements of $\mathfrak{M}$ either as g-modules or as $\Gamma$-modules. If $M^{\prime} \in \mathfrak{M}^{\prime}$ and $M^{\prime \prime} \in \mathfrak{M}^{\prime \prime}$, then $M^{\prime} M^{\prime \prime} \in \mathfrak{M}$, and conversely every element of $\mathfrak{M}$ can be expressed, uniquely, as such a product.

Proof. Assume $M^{\prime} \in \mathfrak{M}^{\prime}$ and $M^{\prime \prime} \in \mathfrak{M}^{\prime \prime}$.

(1) $M^{\prime}$ restricted to $a_{1}$ is irreducible. If $M_{0}$ is the space spanned by those monomials 2.8 for which all $r_{i}$ are short, we show first that $M_{0}=M^{\prime}$. Let $r$ be a long positive root. Then $X_{r} M_{0} \subseteq M_{0}$ because $g_{1}$ is an ideal and $X_{r} v_{+}=0$. We use induction on the height of $r$ to show that $X_{-r} M_{0} \subseteq M_{0}$. This is so if $r$ is simple since then $X_{-r} v_{+}=0$ because $\lambda(r)=0$. If $r$ is not simple, there is a simple root $a$ such that $(r, a)<0$. If $a$ is long, we may write $X_{-r}= \pm\left[X_{-a}\right.$, $\left.X_{-(r-a)}\right]$ and use induction. If $a$ is short, and $w$ denotes the corresponding Weyl reflection and also a corresponding element of $\Gamma$, we may apply the induction hypothesis to the module $M^{\prime w}$ (see 4.1) with high vector $X_{-a}^{\lambda(a)} v_{+}$(see 4.3(a)) and with $M_{0}^{w}$ defined accordingly to get $X_{-w r} \cdot M_{0}^{w} \subseteq M_{0}^{w}$, that is, $X_{-r} M_{0}^{w} \subseteq M_{0}^{w}$. By $4.3(\mathrm{a})$ we have $M_{0}^{w}=M_{0}$. Thus $X_{-r} M_{0} \subseteq M_{0}, M_{0}$ is a g-submodule of $M^{\prime}$, and since $M^{\prime}$ is irreducible, $M_{0}=M^{\prime}$. Next if $v=v_{0}+v_{1}+$ $\cdots+v_{d}$ with $v_{i}$ of height $-i$ and $v_{d} \neq 0$, we prove by induction on $d$ that the $\mathrm{g}_{1}$-module generated by $v$ contains $v_{+}$. If $d>0, X_{r} v \neq 0$ for some $r>0$ by 2.7. By the induction hypothesis, $X_{r_{1}} \cdots X_{r_{k}} X_{r} v=c v_{+}, c \neq 0$, for a sequence $r_{1}, r_{2}, \ldots, r_{k}$ of short roots. Thus $\sum_{l=1}^{k} X_{r_{1}} \cdots\left[X_{r_{i}}, X_{r}\right] \cdots X_{r_{k}} v+X_{r} X_{r_{1}} \cdots$ $X_{r_{k}} v=c v_{+}$. If a term in the sum is nonzero we are done, while if the last term on the left is nonzero we may finish by imitating the last part of the proof that $M_{0}=M^{\prime}$ to show that if $r>0$ and $X_{r} v^{\prime}=v_{+}$then $v_{+}$is in the $g_{1}$ module generated by $v^{\prime}$. By the two parts above, an arbitrary nonzero element of $M^{\prime}$ generates $M^{\prime}$ as $g_{1}$-module, which is (1).

(2) $M^{\prime \prime}$ restricted to $g_{1}$ is 0 . Let $M^{*}$ be the $g\left(\Sigma^{*}\right)$-module in $\mathfrak{M}^{\prime}\left(\Sigma^{*}\right)$ with high weight $\lambda^{*}$ given in terms of the high weight $\lambda$ of $M^{\prime \prime}$ by $\lambda^{*}\left(a^{*}\right)=\lambda(\mathrm{a})$. We may convert $M^{*}$ into a $g\left(\sum\right)$-module by the rule $X . v=(\bar{\theta} X) v\left(X \in g\left(\sum\right)\right.$, $\left.v \in M^{*}\right)$. As such it is irreducible by (1) and the definition of $\bar{\theta}$, is restricted, and has high weight $\lambda$. Thus by 2.7 it is equivalent to $M^{\prime \prime}$. From the definition of $\bar{\theta}, X_{r} . v=0$ if $r$ is short, which is (2). 
(3) Proof of 11.1 for g-modules. Choose a nonzero $u=\sum u_{i}^{\prime} u_{i}^{\prime \prime}$ with the $u_{i}^{\prime}$ linearly independent in $M^{\prime}$ and the $u_{i}^{\prime \prime}$ in $M^{\prime \prime}$. By (1) and (2) we can multiply $u$ by a sequence of $X_{r}(r$ short, $r>0)$ to get a nonzero $v=v_{+}^{\prime} v^{\prime \prime}$, and then $v$ by a sequence of $X_{r}(r$ long, $r>0)$ to get a nonzero multiple of $v_{+}^{\prime} v_{+}^{\prime \prime}$. Using negative roots instead, first short ones and then long ones, we see that this last vector generates $M^{\prime} M^{\prime \prime}$ : we first get all $v^{\prime} v_{+}^{\prime \prime}\left(v^{\prime}\right.$ monomial in $\left.M^{\prime}\right)$, and then using induction on the height of $v^{\prime}$, all $v^{\prime} M^{\prime \prime}$, hence $M^{\prime} M^{\prime \prime}$, which is thus irreducible. Since it is also restricted, it is in $\mathfrak{M}$. Since the high weight of $M^{\prime} M^{\prime \prime}$ is the sum of those of $M^{\prime}$ and $M^{\prime \prime}$, the uniqueness and completeness in 11. 1 follow immediately.

(4) Proof of 11.1 for $I$-modules. As is easily verified, the process 4.2 of lifting the modules of $\mathfrak{M}$ from $g$ to $\Gamma$ is consistent with the two types of tensor products-for algebras and for groups. Thus (4) follows from (3).

11.2 Corollary. If $\left(\sum, p\right)$ is special, the map $\bar{\theta}$ (resp. $\theta$ ) puts the algebra modules (resp. group modules) of $\mathfrak{M}^{\prime \prime}\left(\sum\right)$ in one-one correspondence with those of $\mathfrak{M}^{\prime}\left(\Sigma^{*}\right)$.

In case $(\Sigma, p)$ is special, 11.1 and 11.2 lead to corresponding refinements of $5.1,6.1$ and 7.4 with $\mathfrak{M}$ replaced by $\mathfrak{M}^{\prime}$ and $\mathfrak{M}^{\prime \prime}$. However, there does not seem to be a refinement of the semisimple classes of elements in 7.3. Passing on to $8.2,8.3$ and 8.4 , we have :

11. 3 Corollary. Assume that $\left(\sum, p\right)$ is special. Let $\omega^{\prime}\left(\omega^{\prime \prime}\right)=\Pi \omega_{a}$, the product over the short (long) simple roots, let $l^{\prime}\left(l^{\prime \prime}\right)$ be the number of short (long) simple roots, and let $m^{\prime}\left(m^{\prime \prime}\right)$ be the uumber of short (long) positive roots. Then (a) the $\Gamma_{q^{-}}$module $M_{q}^{\prime}\left(M_{q}^{\prime \prime}\right)$ with high weight $\omega^{q^{-1}}\left(\omega^{\prime \prime q^{-1}}\right)$ has dimension $q^{m^{\prime}}\left(q^{m^{\prime \prime}}\right)$ and character at a semisimple element $h$ given by $\%(h)$ $= \pm q^{d(x)}$ with $2 d(x)+l^{\prime \prime}\left(l^{\prime \prime}\right)$ the dimension of the set of fixed points of $x$ on $\mathrm{g}_{1}\left(\mathrm{~g} / \mathrm{g}_{1}\right)$. (b) The $\Gamma_{q}$-module $M_{p}^{\prime}\left(M_{p}^{\prime \prime}\right)$ has dimension $p^{m^{\prime}}\left(p^{m^{\prime \prime}}\right)$ and a basis consisting of all monomials 2.8 corresponding to short (long) roots $r_{i}$.

The proofs are as in $\S 8$ and will be omitted. To supplement 11.3 we remark that $M_{q}=M_{q}^{\prime} M_{q}^{\prime \prime}$, that $M_{q}^{\prime}$ is quite similar to the corresponding module for the group $\Gamma$ of characteristic 0 (it is of the same dimension, by Weyl's formula), that $M_{q}^{\prime \prime}$ in contrast has lower dimension, that $M_{q}^{\prime}$ for $\sum$ of type $B_{l}$ 
and $q=2$ is just the spin module $\left[13\right.$, p. 20-04] of dimension $2^{l}$, and that $m^{\prime} / m^{\prime \prime}=l^{\prime} / l^{\prime \prime}$ in all cases $[18$, p. 501$]$.

\section{$\S 12$. Twisted groups}

In this, the last, section we extend our results to the finite simple groups associated with the names of Suzuki [22] and Ree [11]. These are defined as follows. A system $\sum$ of type $C_{2}, F_{4}$ or $G_{2}$ may be identified with its dual $\Sigma^{*}$ in such a way that the map $r \rightarrow r^{*}$ of $\sum$ on $\Sigma^{*}$ (see $\S 10$ ) yields an involutary map, $\sigma$, on $\sum$ such that positivity and simplicity of roots is preserved, angles are preserved, but short and long roots are interchanged. If also $\left(\sum, p\right)$ is special, $K$ is algebraically closed, and $k \geq 1$, the isomorphism $\theta$ of 10.1 (c) accordingly yields an automorphism of $\Gamma$ which combines with the $p^{k}$ th power map to produce an automorphism, also denoted $\sigma$, such that

$$
x_{r}(t)^{\sigma}=x_{\sigma r}\left(t^{p s}\right), x_{\sigma r}(t)^{\sigma}=x_{r}\left(t^{s}\right) \quad\left(r \text { short, } s=p^{k}\right) .
$$

If now $n=2 k+1$ and $q=p^{n}$, the fixed points of $\sigma$ form the sought simple group, to be denoted $\Gamma_{q}^{1}$, a subgroup of $\Gamma_{q}$. Observe (see 11.3) that $l^{\prime}=l^{\prime \prime}$ and $m^{\prime}=m^{\prime \prime}$ here.

12.2 Theorem. If $\alpha_{i}$ denotes the $p^{i}$ th power map, every irreducible $\Gamma_{q}^{1}$-module can be written uniquely as $\Pi_{i=0}^{n-1} M_{i}^{\alpha_{i}}\left(M_{i} \in \mathfrak{M}^{\prime}\right)$. If the rank of $\sum$ is $l$, there are $q^{l / 2}$ such modules. Each can be realized over $F_{q}$.

Proof. If $r>0$ and $t \in F_{q}, \Gamma_{q}^{1}$ contains an element of the form $x=$ $x_{r}(t) x_{o r}\left(t^{p s}\right) \Pi x_{r^{\prime}}\left(t^{\prime}\right)$, the product over roots $r^{\prime}$ which are positive integral linear combinations of $r$ and $\sigma r$ (proof by downward induction on the height of $r$ ). Thus if $v \in M \in \mathfrak{M}^{\prime}$ and $v$ is homogeneous,

$$
(x-1) v=t X_{r} v+t^{p s} X_{\sigma r} v+\text { higher terms. }
$$

If we refine the notion of height so that a positive linear combination of simple roots is taken to be lower than another one of the same height as previously defined if fewer short simple roots are used, then $r$ is lower than $o r$. In fact, if $r=\sum[c(a) a+d(a) \sigma a]$, the sum over the short simple roots $a$, then or $=\sum\left[p d(a) a+c(a)_{\sigma a}\right]:$ since $\sigma$ preserves angles, the map $r^{\prime} \rightarrow p^{-1 / 2} \sigma r^{\prime}, \sigma r^{\prime} \rightarrow$ $p^{1 / 2} r^{\prime}$ ( $r^{\prime}$ short) comes from an isometry, which maps $r$ to $\sum\left[p^{-1 / 2} c(a)_{\sigma a}+\right.$ $\left.p^{1 / 2} d(a) a\right]=p^{-1 / 2} \sum\left[p d(a) a+c(a)_{\sigma a}\right]$. With a corresponding refinement in the 
notion of homogeneity, we thus get 12.3 with the second term of the right missing, and a similar equation for negative roots. Combining these equations with the key fact, proved as $11.1(1)$, that each $M \in \mathfrak{M}^{\prime}$ is irreducible as $\mathfrak{g}_{1}$ module, we can now prove, almost exactly as in 5.1 , that $\Pi M_{i}^{\alpha^{i}}\left(M \in \mathfrak{D}^{\prime}\right)$ is irreducible. The proof that this product determines its components $M_{i}$ can also be taken from 5.1. As for completeness of the set of $q^{l / 2}$ product modules, the semisimple classes of $\Gamma_{q}^{1}$ are characterized by coordinates $\gamma(a)$ and $\gamma(\sigma a)$ $\left(a \in S, a\right.$ short) for which $\gamma(a)^{p s}=\gamma(\sigma a)$ and $\gamma(\sigma a)^{s}=\gamma(a)$ (see 3.9 and 12.1); their number is thus $q^{l / 2}$, as required. Finally, as the restriction of a $\Gamma_{q}$-module, each irreducible $I_{q}^{1}$-module can be realized over $F_{q}$ by 7.5 .

As a supplement to 12.2 we have:

12.4. For $\sum$ of type $C_{2}$ or $F_{4}$ and $p=2$, every irreducible projective representation of $\Gamma_{q}^{1}$ can be lifted to an ordinary one.

This result, proved in [21], quite likely also holds for the remaining case, $\sum$ of type $G_{2}$ and $p=3$.

Also since the axioms of [17] are easily verified for the groups $\Gamma_{q}^{1}$ there is an analogue of 8.1 , and modifying the development of $\S 8$, we have no trouble in proving:

12.5 Theorem. (a) $\Gamma_{q}^{1}$ has an irreducible module $M_{q}^{\prime}$, constructed by the methods of [17], of dimension $q^{m / 2}$, the order of a Sylow group in $\Gamma_{q}^{1}$ (here $m$ is the number of positive roots in $\Sigma$ ). (b) $M_{q}^{\prime}$ is equivalent to the restriction to $\Gamma_{q}^{1}$ of the $\Gamma_{q}$-module $M_{q}^{\prime}$ of $11.3 . \quad$ (c) All other irreducible $\Gamma_{q}^{1}$-modules have lower dimensions than $M_{q}^{\prime}$.

From 12.2 we know all irreducible $\Gamma_{q}^{1}$-modules (in fact all $\Gamma_{q}$-modules also by $7.4,11.1$ and 11.2 ) once we know those in $\mathfrak{M}^{\prime}$. We consider the individual cases. For $\sum$ of type $C_{2}, p^{l / 2}=2$, so that the trivial 1-dimensional module and the 4-dimensional module $M_{2}^{\prime}$ of $11.3(\mathrm{~b})$, on which $\Gamma_{q}$ acts as the symplectic group, exhaust $\mathfrak{M}^{\prime}$. For $\sum$ of type $G_{2}, p^{l / 2}=3$, we have in $\mathfrak{M}^{\prime}$ the trivial module, the module $g_{1}$, of dimension 7 , and the module $M_{3}^{\prime}$ of $11.3(\mathrm{~b})$, of dimension $3^{3}=27$. Finally, for $\sum$ of type $F_{4}, p^{l / 2}=4$, there are the trivial module, the module $g_{1}$, of dimension 26 , and the module $M_{2}^{\prime}$, of dimension $2^{12}=4096$, leaving one module yet to be described. 


\section{RefERENCES}

[1] Brauer, R. and Nesbitt, C., On the modular representations of groups of finite order, U. of Toronto Studies (1937), 1-21.

[2] Brauer, R. and Nesbitt, C., On the modular characters of groups, Ann. of Math. 42 (1941), 556-590.

[ 3 ] Chevalley C., Sur certains groupes simples, Tôhoku Math. J. 7 (1955), 14-66.

[4] Curtis, C. W., Representations of Lie algebras of classical type with applications to linear groups, J. Math. and Mech. 9 (1960), 307-326.

[5] Curtis, C. W., On projective representations of certain finite groups, Proc. Amer. Math. Soc. 11 (1960), 852-860.

[6] Curtis, C. W., On the dimensions of the irreducible modules of Lie algebras of classical type, Trans. Amer. Math. Soc. 96 (1960), 135-142.

[ 7 ] Dickson, L. E., The abstract form (two papers), Quart. J. Math. 38 (1907), 141-158.

[ 8 ] Hertzig, D., Forms of algebraic groups, Proc. Amer. Math. Soc. 12 (1961), 657-660.

[ 9 ] Lang, S., Algebraic groups over finite fields, Amer. J. Math. 78 (1956), 555-563.

[10] Mark, C., Thesis, U. of Toronto.

[11] Ree, R., A family of simple groups associated with the simple Lie algebra of type $\left(F_{4}\right)$, second paper: $\left(G_{2}\right)$, Amer. J. Math. 83 (1961), 401-420, 432-462.

[12] Rosenlicht, M., Some rationality questions on algebraic groups, Ann. di Mat. 43 (1957), $25-50$.

[13] Séminaire C. Chevalley, Classification des Groupes de Lie Algébriques (two volumes), Paris (1956-8).

[14] Séminaire "Sophus Lie," Paris (1954-5).

[15] Serre, J. P., Groupes algébtiques et corps de classes, Hermann, Paris (1959).

[16] Steinberg, R., A geometric approach, Trans. Amer. Math. Soc. 71 (1951), 274-282.

[17] Steinberg, R., Prime power representations of finite linear groups II, Can. J. Math. 9 ( 1957), 347-351.

[18] Steinberg, R., Finite reflection groups, Trans. Amer. Math. Soc. 91 (1959), 493-504.

[19] Steinberg, R., Variations on a theme of Chevalley, Pacific J. Math. 9 (1959), 875-891.

[20] Steinberg, R., The simplicity of certain groups, Pacific J. Math. 10 (1960), 1039-1041.

[21] Steinberg, R., Générateurs, relations et revêtements de groupes algébriques, Colloque sur la théorie des groupes algébriques, Bruxelles (1961).

[22] Suzuki, M., On a class of doubly transitive groups, Ann. of Math. 75 (1962), 105-145.

[23] Tits, J., Sur les analogues algébriques des groupes semisimple complexes, Colloque d'algèbre supérieure, Bruxelles (1956), 261-289.

[24] Tits, J., Les "formes réelles" des groupes de type $E_{6}$, Séminaire Bourbaki 162, Paris (1958).

[25] Tits, J., Sur la trialité et certains groupes qui s'en déduisent, Paris Inst. Hautes Etudes Sci. Publ. Math. 2 (1959), 37-84.

[26] Weyl, H., Theorie der Darstellung kontinuierlicher halb-einfacher Gruppen durch lineare Transformationen III, Math. Zeit (1926), 377-395.

[27] Wong, W. J., Thesis, Harvard U.

Institute for Advanced Study, Princeton

University of California, Los Angeles 